Revue d'histoire de l'Amérique française

QU. REVUE D.HISTOIRE DE L'AMÉRIQUE FRANÇAISE

\title{
Les voyages outre-mer d'un nom : de Loreto en Italie à la Jeune-Lorette au Canada
}

\section{Karin Vélez}

Volume 64, numéro 3-4, hiver-printemps 2011

La Nouvelle-France et l'Atlantique

URI : https://id.erudit.org/iderudit/1017972ar

DOI : https://doi.org/10.7202/1017972ar

Aller au sommaire du numéro

\section{Éditeur(s)}

Institut d'histoire de l'Amérique française

\section{ISSN}

0035-2357 (imprimé)

1492-1383 (numérique)

Découvrir la revue

\section{Citer cet article}

Vélez, K. (2011). Les voyages outre-mer d'un nom : de Loreto en Italie à la Jeune-Lorette au Canada. Revue d'histoire de l'Amérique française, 64(3-4), 119-144. https://doi.org/10.7202/1017972ar

\section{Résumé de l'article}

$\mathrm{Au} \mathrm{XVII}{ }^{\mathrm{e}}$ siècle, des centaines de chapelles, hors du territoire de l'Italie, reçurent le nom de Loreto en mémoire de la célèbre basilique mariale sur la côte adriatique italienne. L'attribution de noms est souvent associée à l'occupation impériale ou culturelle. Mais j'adopte ici une autre position. Je démontre plutôt que les catholiques de différents empires et de cultures différentes s'inspirèrent spontanément du nom Loreto pour établir une confrérie au-delà des mers. Leurs réseaux improvisés révèlent un monde de l'époque moderne bâti sur des frontières poreuses et sur un terrain d'entente plutôt que sur la rigidité impériale monolithique. Dans cet article, j'étudie trois groupes dissemblables de passeurs autodésignés du nom Loreto : les marchands picènes de la région des Marches, en Italie, où se trouve la basilique ; des missionnaires jésuites français et espagnols qui ont dédié des chapelles à Notre-Dame de Lorette, ou Loreto, au Canada et au Mexique, respectivement ; et des convertis hurons de la mission de Lorette, près de Québec. Le catholicisme qu'ils partageaient rendait familiers les symboles étrangers et resituait ces acteurs dans le cadre d'un empire français (catholique). Pourtant, ces échanges compliquent aussi l'idée d'un monde atlantique "français ", puisqu'ils montrent des catholiques du XVII ${ }^{\mathrm{e}}$ siècle qui vont au-delà de leurs collectivités - italienne, française ou huronne - pour bâtir une communauté mondiale de frères et de soeurs dans la religion.
Tous droits réservés @ Institut d’histoire de l'Amérique française, 2013

Ce document est protégé par la loi sur le droit d'auteur. L'utilisation des services d'Érudit (y compris la reproduction) est assujettie à sa politique d'utilisation que vous pouvez consulter en ligne.

https://apropos.erudit.org/fr/usagers/politique-dutilisation/ 


\title{
Les voyages outre-mer d'un nom : de Loreto en Italie à la Jeune- Lorette au Canada
}

\author{
KARIN VÉLEZ \\ Macalester College
}

RÉSumÉ • Au XVIIe siècle, des centaines de chapelles, hors du territoire de l'Italie, reçurent le nom de Loreto en mémoire de la célèbre basilique mariale sur la côte adriatique italienne. L'attribution de noms est souvent associée à l'occupation impériale ou culturelle. Mais j'adopte ici une autre position. Je démontre plutôt que les catholiques de différents empires et de cultures différentes s'inspirèrent spontanément du nom Loreto pour établir une confrérie au-delà des mers. Leurs réseaux improvisés révèlent un monde de l'époque moderne bâti sur des frontières poreuses et sur un terrain d'entente plutôt que sur la rigidité impériale monolithique. Dans cet article, j'étudie trois groupes dissemblables de passeurs autodésignés du nom Loreto: les marchands picènes de la région des Marches, en Italie, où se trouve la basilique; des missionnaires jésuites français et espagnols qui ont dédié des chapelles à Notre-Dame de Lorette, ou Loreto, au Canada et au Mexique, respectivement; et des convertis hurons de la mission de Lorette, près de Québec. Le catholicisme qu'ils partageaient rendait familiers les symboles étrangers et resituait ces acteurs dans le cadre d'un empire français (catholique). Pourtant, ces échanges compliquent aussi l'idée d'un monde atlantique «français», puisqu'ils montrent des catholiques du XVIle siècle qui vont au-delà de leurs collectivités - italienne, française ou huronne - pour bâtir une communauté mondiale de frères et de sœurs dans la religion.

ABSTRACT • In the 1600s, hundreds of chapels outside Italian jurisdiction were named «Loreto» after the famed Marian shrine on Italy's Adriatic coast. Nomenclature is often associated with imperial or cultural occupation. But I argue otherwise here. I demonstrate instead that Catholics of different empires and cultures spontaneously drew on the designation «Loreto » to establish brotherhood across the ocean. Their improvised linkages uncover 
an early modern world built on porous boundaries and common ground rather than monolithic imperial rigidity. In this paper, I consider three disparate groups of self-appointed Loreto networkers: Picene merchants of the Marche region of Italy, home to the namesake shrine; French and Spanish Jesuit missionaries who dedicated chapels to Loreto in Canada and Mexico, respectively; and Huron converts of the Lorette mission near Québec City. Shared Catholicism made foreign symbols familiar, resituating these actors within the bounds of a French (Catholic) empire. Yet these exchanges also complicate the idea of a «French» Atlantic, since they reveal seventeenth-century Catholics self-consciously reaching beyond Italian, French, and Huron ranks to build a global community of religious brethren.

C omment les lieux reçoivent-ils leur nom? Dans le cas de la basilique vieille de sept cents ans de Loreto, en Italie, la réponse semble étrangement accidentelle ${ }^{1}$. Le récit en est fait par Girolamo Angelitta, secrétaire de la ville de Recanati, voisine de Loreto, dans la région italienne des Marches. Dans l'histoire de sa basilique, qu'il écrivit au $\mathrm{XVI}^{\mathrm{e}}$ siècle, Angelitta raconte comment, le 2 décembre 1295, une petite maison tomba du ciel et atterrit au milieu de la route qui menait à Recanati ${ }^{2}$. Elle était posée là, au sommet d'une colline boisée, non loin de l'Adriatique qui bordait la propriété d'une femme de Recanati prénommée Laureta. Ce prénom fut plus tard attribué à la mystérieuse maison et à la Madone noire qu'elle abrita.

Selon Angelitta, on ne tarda pas à établir que cette Sainte Maison de Loreto était la véritable maison de la Vierge Marie, transportée miraculeusement par des anges depuis Nazareth jusqu'en Italie. En comparaison de son arrivée spectaculaire, la prosaïque attribution à cette maison du nom d'une femme des environs paraît banale, presque décevante. L'impression se renforce lorsqu'on considère que la relique est passée par plusieurs endroits avant Loreto, et qu'elle aurait facilement pu accumuler et conserver d'autres noms pour commémorer ses voyages. Angelitta rapporte que la Sainte Maison s'est arrêtée quatre fois avant d'atteindre Loreto. Les habitants du premier arrêt ont entretenu des liens avec la relique pendant des siècles après qu'elle les eut abandonnés.

Après avoir quitté la Terre sainte, apparemment, la Santa Casa de Loreto atterrit tout d'abord sur une colline près du village de Trsat, aux limites

1. Je remercie tout particulièrement Catherine Desbarats, Thomas Wien et le Groupe d'histoire de l'Atlantique français qui m'ont offert la possibilité de présenter le contenu de cet article dans le cadre du colloque Des Mondes Atlantiques Français à Montréal, en octobre 2009. Mes remerciements s'adressent également aux évaluateurs anonymes de l'article pour les suggestions utiles qu'ils m'ont envoyées.

2. Girolamo Angelitta, L'historia della Traslatione della Santa Casa della Madonna a Loreto (Macerata, Sebastiano Martorelli, 1580). 
de ce qui est aujourd'hui la ville côtière de Rijeka, en Croatie. À l'époque, plusieurs noms servaient à désigner cette région: Dalmatie, Illyrie, Slavonie. Mais la Sainte Maison n'y resta que trois ans avant de se déraciner de nouveau. En quittant les lieux, elle abandonna le nom "Trsat», tout comme elle avait laissé «Nazareth» derrière elle. Cette fois, la relique catholique tomba sur la rive opposée de l'Adriatique où, après deux autres déplacements, elle finit par se déposer à un endroit qu'occupait déjà une Italienne ordinaire, Laureta, qui vaquait à ses affaires, tout comme la jeune Vierge Marie l'avait fait. Pourtant, ce fut le nom de Laureta - et non un nom transplanté, importé, exotique, ni même un nom biblique, mais un nom local - qui resta associé au sanctuaire.

Je commence par la mythohistoire de la basilique mariale la plus célèbre d'Italie, parce qu'elle renverse parfaitement un présupposé très répandu concernant les noms aux époques de rencontres interculturelles: un présupposé de territorialité. Instinctivement, nous cherchons à découvrir la personne ou le groupe dominant qui s'est avancé sur des terres nouvelles et a imposé un nom. Donner un nom est souvent associé avec la conquête et peut être interprété comme un geste de pouvoir ou une affirmation autoritaire de sa présence ${ }^{3}$. C’est le cas en particulier de l'époque moderne, colorée par les récits de l'expansion européenne et catholique. Aux XVII et XVIII ${ }^{e}$ siècles, la période qui nous concerne ici, nommer semble souvent n'être, pour les Européens, qu'une "cérémonie de prise de possession" du Nouveau Monde au même titre que les autres ${ }^{4}$. De même que les haies anglaises, les processions françaises, les discours espagnols, les cartes hollandaises et les cartes du ciel portugaises, l'attribution d'un nom joue un rôle de proclamation de l'appartenance, à une échelle réduite, mais offensive. Même - et en particulier - à cette époque expansionniste, le nom Loreto paraît défier toute attente. Il apparaît par hasard, arrive avant et sans la Sainte Maison, ou traîne quelque part sans raison, exigeant que nous tracions un portrait plus nuancé de la manière dont les noms (et les dévotions catholiques qui y sont associées) se déplacent et pourquoi ils persistent.

3. Sur l'attribution de noms en tant qu'appropriation intentionnelle associée à la conquête, voir Stephen Greenblatt, Marvelous Possessions: The Wonder of the New World (Oxford, Clarendon Press, 1991), 57-58. Sur les précédents survenus en Europe de recours à des noms pour « déposséder» et prendre possession de nouveaux territoires, voir Fabienne L. Michelet, Creation, Migration, and Conquest: Imaginary Geography and Sense of Space in Old English Literature (New York, Oxford University Press, 2006), 266.

4. Patricia Seed, Ceremonies of Possession in Europe's Conquest of the New World, 1492-1640 (New York, Cambridge University Press, 1995). 

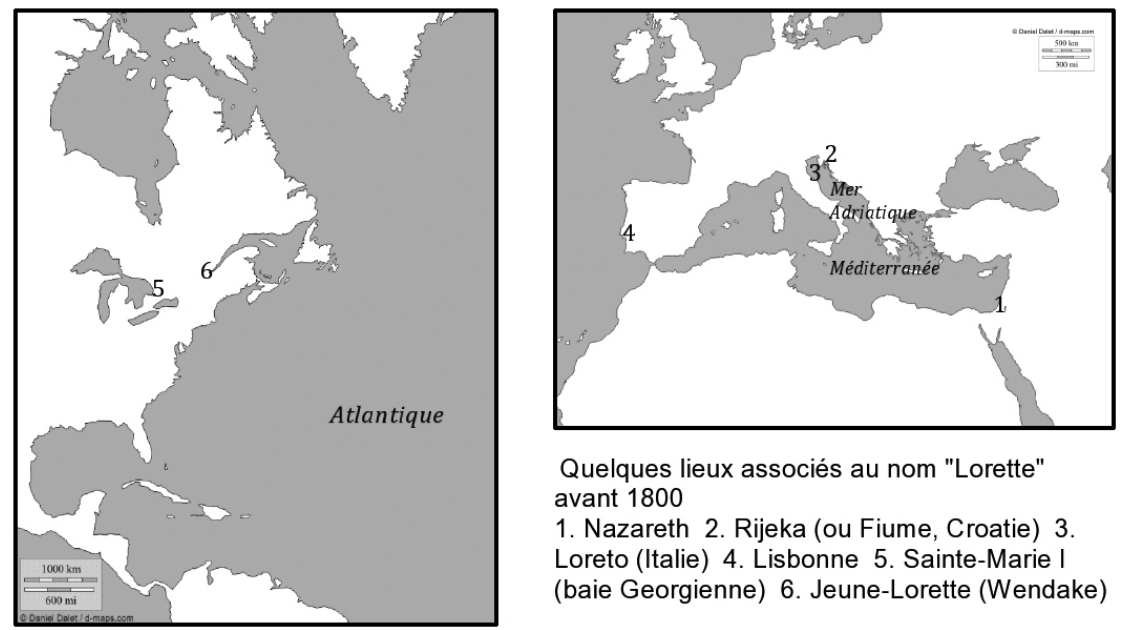

Quelques lieux associés au nom "Lorette" avant 1800

1. Nazareth 2. Rijeka (ou Fiume, Croatie) 3. Loreto (Italie) 4. Lisbonne 5. Sainte-Marie I (baie Georgienne) 6. Jeune-Lorette (Wendake)

J'explore ici les méandres de la nomenclature catholique en suivant Loreto dans son cheminement de nom de l'arrière-pays qui devient un marqueur de l'identité italienne et ensuite une référence internationalement reconnue du catholicisme dans son ensemble. Je commence brièvement par l'Italie, où je m'intéresse aux marins qui s'embarquent des ports situés près de Loreto. Je traverserai ensuite l'Atlantique pour examiner une transplantation francophone et une transplantation huronne de Loreto: la première à l'initiative d'un jésuite français, Pierre-Joseph-Marie Chaumonot, et la deuxième à celle des Hurons de Québec, nouveaux convertis au catholicisme qui se battirent pour garder vivants leurs liens avec la dévotion lorettaine au milieu du XVIII ${ }^{\mathrm{e}}$ siècle ${ }^{5}$. Que signifiait Loreto pour ce groupe disparate de catholiques italiens, français et hurons? Pourquoi ces groupes ont-ils choisi séparément de transporter ce nom avec eux et de le poser en de nouveaux endroits? Le nom «Lorette» est peut-être déjà familier aux lecteurs de l'histoire nord-américaine, mais quand on le replace dans son contexte plus large, transatlantique, il importe de rappeler qu'à cette époque, les marqueurs catholiques ne restaient pas fixés en permanence à

5. L'observation remarquablement documentée d'André Sanfaçon, selon laquelle les Jésuites ont été les passeurs de la dévotion italienne de Loreto hors de l'Europe, jusqu'en Nouvelle-France et ailleurs, m'a servi d'inspiration dans mes recherches. Je réagis directement, ici, à son appel à la contextualisation de cette diffusion, plutôt que de la traiter comme un "phénomène isolé» (p. 216); je me concentre en outre sur le nom «Loreto ", plutôt que sur l’artefact de la Sainte Maison. André Sanfaçon, «"A New Loreto in New France" : Pierre-Joseph-Marie Chaumonot, S.J., and the Holy House of Loreto ", dans Germaine Warkentin et Carolyn Podruchny, dir., Decentering the Renaissance : Europe in Multidisciplinary Perspective, 1500-1700 (Toronto, University of Toronto Press, 2001), 200-220. 
des coins de pays ou à des colonisateurs, que ce soit en Europe ou ailleurs. Cette approche transocéanique met en lumière les convergences et les divergences du catholicisme de part et d'autre des nombreuses frontières - spatiales, politiques et culturelles - qui quadrillaient le monde atlantique de l'époque moderne ${ }^{6}$.

\section{DES NOMS LOCAUX: LES MARCHANDS PICÈNES}

Les marins issus du territoire environnant la basilique de Loreto furent les premiers à introduire leur madone locale partout où ils voyageaient. Par la suite, la désignation apparut en des lieux éloignés de leurs circuits méditerranéens, dérivant avec les pèlerins et grâce aux répons maintes fois répétés des litanies de Loreto colligées par le jésuite allemand Peter Canisius en $1558^{7}$. Mais toute étude sur les invocations lorettaines à l'étranger doit commencer en amont des populaires litanies, par les habitants, impétueux et grands voyageurs, de la région des Marches, les Picènes.

«Picène» est un terme romain archaïque, encore utilisé communément au XVIII ${ }^{\mathrm{e}}$ siècle pour désigner les habitants de la région côtière du sud-est de l'Italie, où Loreto est situé. L'histoire ancienne de cette région met en scène des peuples autochtones, des militaires commerçants et des frontières contestées, signes avant-coureurs des événements et des personnages qui se produiront sur la frange pionnière de la Nouvelle-France du XVII ${ }^{e}$ siècle. Piceni était le nom romain donné aux peuples établis le long de la côte est de l'Italie au III ${ }^{\mathrm{e}}$ siècle avant l'ère chrétienne; les Romains étendirent leur occupation et leur administration jusqu'à cette frontière que constituait la mer Adriatique et des envahisseurs francs succédèrent aux Romains au VIII ${ }^{e}$ siècle de l'ère chrétienne. La région fut alors connue comme étant celle des Marches, en référence au terme francique désignant une région frontalière, marka. Ainsi, très tôt dans l'histoire de Loreto, les noms et les peuples empiétaient déjà les uns sur les autres, témoignant d'un espace contesté.

Pour les besoins de cet article, le nom «Picènes» servira à décrire les autochtones de quatre provinces italiennes côtières, chacune correspondant grosso modo à l'une des villes bien établies au XVII siècle que nous énumérons ici du nord au sud: Pesaro-Urbino, Ancône, Macerata et Ascoli

6. Allan Greer et Kenneth Mills, "A Catholic Atlantic», dans Jorge Cañizares-Esguerra et Erik R. Seeman, dir., The Atlantic in Global History,1500-2000 (Upper Saddle River, Prentice Hall, 2007), 3-19.

7. Jeffrey Chipps Smith, Sensuous Worship: Jesuits and the Art of the Early Catholic Reformation in Germany (Princeton, Princeton University Press, 2002), 143-154. 
Piceno. Loreto se trouvait entre deux de ces grandes villes, le port d'Ancône au nord et la ville de Macerata à l'intérieur des terres, au sud-ouest. Compte tenu de la situation centrale de Loreto dans le territoire traditionnel des Picènes, les marins des Marches la considéraient comme leur propre basilique. Aux mains de ces hommes de la mer, Loreto commença à larguer ses amarres.

Lorsque les marins picènes devaient demeurer dans des ports étrangers, ils se regroupaient fréquemment autour de confréries mariales dédiées à la Vierge de Loreto. On peut faire le rapprochement avec les marchands portugais qui rassemblaient leur collectivité dispersée autour d'un des saints de leur pays, saint Antoine (1195-1231). Les commerçants portugais vivaient dispersés sur le pourtour de l'Atlantique au XVII siècle. Dans les villes portuaires, en pleine mer ou de l'autre côté, au Brésil, les expatriés portugais se rassemblaient autour d'Antoine, l'humble prédicateur franciscain du XIII ${ }^{\mathrm{e}}$ siècle né dans le grand port maritime du Portugal, Lisbonne $^{8}$. Bien que saint Antoine ait fini ses jours à Padoue, en Italie, ville où ses restes sont conservés aujourd'hui et qui le revendique comme sien, les Portugais considéraient Antoine comme leur saint patron en raison de ses origines lisboètes".

Tout comme les Portugais regroupèrent leurs communautés autour de saint Antoine, ainsi agirent les Picènes avec la Madone de Loreto. Un groupe d'immigrants picènes établis à Rome en offre un exemple typique. En 1670, ils occupèrent l'église de San Salvatore in Lauro, située dans leur voisinage et abandonnée depuis peu: ils en firent un lieu de dévotion lorettaine ${ }^{10}$. Ils trouvèrent que les noms étaient significatifs et que cette coïncidence ne pouvait pas mieux tomber. San Salvatore in Lauro et Loreto - il y avait une ressemblance commode entre le nom du nouveau siège de leur confrérie et celui de la basilique de leur ancien coin de pays. San Salvatore in Lauro devint le principal point de rassemblement des voyageurs et des expatriés

8. Ronald Vainfas, «St. Anthony in Portuguese America: Saint of the Restoration», dans Allan Greer et Jodi Bilinkoff, dir., Colonial Saints: Discovering the Holy in the Americas, 1500-1800 (New York, Routledge Press, 2003), 101-102. Antoine est né en 1195, 60 ans avant que Lisbonne devienne la capitale du royaume.

9. Daviken Studnicki-Gizbert, A Nation Upon the Ocean Sea: Portugal's Atlantic Diaspora and the Crisis of the Spanish Empire, 1492-1640 (New York, Oxford University Press, 2007), 56-57.

10. Dès 1638, les Picènes habitant à Rome se réunissaient sous les auspices religieux de la Vierge de Loreto. Il existe des documents concernant une petite confrérie picène qui lui était dédiée et qui se réunissait dans l'église San Michele, dans la Via Ripetta, à Rome. En 1670, ce groupe s'installa dans une autre église, près de leur quartier résidentiel. Cette église, San Salvatore in Lauro, avait été fermée lorsque les clercs de San Giorgio in Alga l'avaient quittée. Les Picènes s'y étaient installés. Voir Maurice De Jonghe, Roma: Santuario Mariano, vol. VII de la série Roma Cristiana, sous la direction de Carlo Galassi Paluzzi, Bologne (Cappelli, 1969), 153-154. 
picènes se trouvant à Rome ${ }^{11}$. En 1716, un chanoine écrivant à propos de l'église de ce quartier la décrivit comme appartenant à «la nation picène ${ }^{12}$ ».

Transplanté loin de la côte picène, cependant, Loreto se chargea d'un nouveau pouvoir d'attraction. En 1676, l'énorme cathédrale de Loreto fut terminée dans le port de Lisbonne. La dévotion lorettaine de Lisbonne avait des racines antérieures à celles de la dévotion de Rome. À partir du $\mathrm{XVI}^{\mathrm{e}}$ siècle, Lisbonne comptait une population appréciable de marchands originaires d'Italie, dont certains étaient venus de l'Adriatique à bord de bateaux baptisés Loreto ${ }^{13}$. Leur nouvelle cathédrale de Loreto se tailla une niche dans la ville portuaire du Portugal en offrant des messes hebdomadaires en italien vernaculaire. À cause de ce service linguistique, la paroisse de Loreto devint un lieu de rassemblement pour tous les italophones de Lisbonne et non seulement pour les Picènes ${ }^{14}$. La cathédrale de Loreto publicisa cette identité augmentée. En 1751, par exemple, un service funèbre à la mémoire du roi João $\mathrm{V}$ du Portugal fut célébré dans cette

11. Lorsqu'on latinise le nom Loreto, on obtient Lauret-, dont la racine est aussi en partie celle de Lauro, mot qui désigne le laurier en latin. Un historien anonyme de la confrérie a commenté cette ressemblance et mentionné que «Lauro " peut soit décrire un laurier qui se trouvait jadis à l'endroit où cette église a été bâtie à Rome, soit correspondre à une addition ultérieure qui ferait allusion à la nouvelle dévotion lorettaine de l'église. Biblioteca Angelica (Rome) Ms. 1610/2, Statuti, folios 1-11.

12. Giovanni Maria Crescimbeni - lui-même Picène, car il était originaire de la ville de Macerata employa ces mots dans le titre de l'ouvrage qu'il publia en 1716 sur cette église de Rome (nous soulignons): Memorie Istoriche della Miracolosa Immagine di S. Maria delle Grazie, Esistente in Roma nella V. Chiesa, detta già S. Salvatore in Lauro, ed ora S. Maria di Loreto della Nazione Picena. Le terme "nation" apparaît dans les documents de la fin du XVII ${ }^{\mathrm{e}}$ siècle et du début du XVIII ${ }^{\mathrm{e}}$ en particulier pour désigner les assemblées de fidèles expatriés italophones et d'origine picène attachés à Loreto. Sur le sentiment flou d'appartenance "nationale» vécu au cours de ces siècles de l'expansion du catholicisme en particulier, voir Barbara Fuchs, "Religion and National Distinction in the Early Modern Atlantic», dans Linda Gregerson et Susan Juster, dir., Empires of God: Religious Encounters in the Early Modern Atlantic (Philadelphie, University of Pennsylvania Press, 2011), 58-69. Sur le «sentiment national» et les loyautés régionales à cette époque, voir David A. Bell, The Cult of the Nation in France: Inventing Nationalism, 16801800 (Cambridge, Harvard University Press, 2003), et William J. Kennedy, The Site of Petrarchism: Early Modern National Sentiment in Italy, France and England (Baltimore, Johns Hopkins University Press, 2003).

13. Les marins italophones de Lisbonne provenaient de diverses régions de l'Italie, mais on peut supposer qu'il y avait des Picènes parmi eux, ce qui expliquerait leur choix de la Madone picène de Loreto comme centre de leur dévotion dans leur nouvelle paroisse. Des navires baptisés «Loreto » et mouillant dans le port de Lisbonne étaient commandés par des Italiens et ils faisaient du commerce au départ de Lisbonne. Il arrivait souvent que des marins baptisent leur navire du nom de la Madone locale. Peut-être que, dans le cas de certains de ces bateaux portant le nom «Loreto», des Picènes les avaient nommés ainsi, en étaient propriétaires ou en formaient l'équipage.

14. Il convient de mentionner que, sur les 51 paroisses catholiques de Lisbonne, Loreto semble être la seule qui soit historiquement associée à un groupe unique d'immigrants. Avant de se diviser entre Portugais et Italiens, la communauté de fidèles semble avoir incité au moins un de ses membres portugais à embrasser la dévotion de Loreto et peut-être, par extension, la Compagnie de Jésus. Bartholomeu Nunes Correa fut baptisé dans l'église de Loreto, à Lisbonne, en 1663 et entra chez les Jésuites en 1681. Voir Arquivo Nacional de Torre do Tombo, Lisbonne (ANTT), Cartório Jesuitico, Maço 83, doc. 176. Voir aussi ANTT, Registos Paroquais, Noticias Históricas sobre as Freguesias de Lisboa [1992], 223, 226. 
église. Le feuillet distribué en souvenir de ces solennités portait la signature de «la nation italienne résidant à Lisbonne ${ }^{15}$ ».

Nous assistons ici à une extension graduelle de Loreto. À l'intérieur de l'Italie, ce terme indiquait la côte des Picènes; au-delà, dans des entrepôts comme Lisbonne, il renvoyait à la totalité de l'expansion linguistique de l'Italie. Les italophones à l'étranger utilisaient de plus en plus une Madone située à la périphérie - Loreto - comme point focal de leur identité commune émergente. De manière significative, dans les deux cas, Loreto ne fut pas imposé exclusivement par des gens venus d'ailleurs. Les Picènes et les italophones eux-mêmes employaient souvent ce mot, afin de se reconnaître et de se faire reconnaître hors de l'Italie. Ces vagabonds adriatiques ont effectivement choisi une référence catholique reconnaissable et les pratiques ecclésiales associées à cette référence afin de légitimer leur présence à Lisbonne, ville catholique. Mais leur choix particulier de la Madone de Loreto comme point focal de leur communauté les ramenait à ce coin de la côte adriatique qu'ils appelaient leur pays. Ce sentiment aigu de régionalisme adriatique est même apparent chez les jésuites qui, les premiers, ont appliqué le nom de Loreto outre-mer.

\section{DES NOMS SUPERPOSÉS: LE JÉSUITE CHAUMONOT, PASSEUR DU NOM LORETO}

Un des premiers jésuites à avoir transporté le nom de Loreto outre-mer fut Pierre Chaumonot. Il le fit en partie en raison d'une visite personnelle à la basilique mariale italienne et en partie à cause des liens solides que son ordre, la Compagnie de Jésus, entretenait avec Loreto. Les Jésuites, extrêmement mobiles, furent d'importants vecteurs dans les périples de cette dévotion adriatique. Ils prenaient souvent de vieux noms catholiques et les conféraient à de nouveaux endroits et à de nouveaux peuples. En tant que jésuite possédant une façon particulièrement réflexive de choisir des noms, Chaumonot exprimait la complexité et la nature cumulative de ce processus. Non seulement a-t-il répandu le nom catholique de Loreto, mais il a délibérément adopté de nouveaux noms pour lui-même. En outre, Chaumonot mettait souvent sur le même pied la désignation des lieux et celle des gens. Cette façon de prendre l'un pour l'autre un nom, un lieu et une personne semble avoir servi à relier les uns aux autres dif-

15. Remarquons le titre complet de ce document conservé à la Biblioteca Nacional, à Lisbonne (nous soulignons): Noticia do apparato, e magnificas disposiçoens, que para as solemnes exequias de Sua Magestade fidelissima o multo augusto Rey do Portugal Dom João V, celebrou na sua Igreja de Nossa Senhora do Loreto em 14 de janeiro de 1751. A Naçam Italiana, residente em Lisboa. S.I., 17??. 
férents groupes de catholiques et à les mettre en rapport avec leurs errances passées. L’expérience de Chaumonot, comme jésuite et comme catholique vivant loin de chez lui, montre tout l'éventail de possibilités qui existaient à l'époque moderne, pour l'action personnelle, la créativité et l'établissement de liens au moyen de la revendication de noms.

Pour comprendre Chaumonot, il faut d'abord revenir aux Jésuites et à leur affinité avec Loreto. Les Jésuites du début du XVII ${ }^{\mathrm{e}}$ siècle étaient prédisposés à penser à ce site de la côte adriatique. Au cours des décennies formatrices de la Société de Jésus, on encourageait tous les aspirants jésuites à faire un pèlerinage à pied dans le cadre de leur formation à l'ascétisme. La basilique mariale de Loreto devint la destination normale de cet exercice, puisque Ignace de Loyola, fondateur de la Compagnie de Jésus, avait favorisé cette destination. En outre, Loreto était bien située, à une semaine de marche de Rome, vers l'est. Ainsi, la plupart des jésuites de la première génération s'étaient rendus à la basilique mariale.

Bon nombre des premiers jésuites étaient italiens. Certains des premiers jésuites à invoquer Loreto à l'étranger venaient de la région picène. Matteo Ricci (1552-1610), qui prit la peine d'introduire en Chine la Madone de Loreto, «se vantait parfois devant les Chinois d'être né près de Loreto, pratiquement à l'ombre de la maison où avait vécu la Vierge Marie ${ }^{16}$ ». En réalité, il était né à Macerata, la ville la plus proche de Loreto, où, quatre ans auparavant, la Vierge de Loreto était miraculeusement apparue à un habitant du lieu ${ }^{17}$. L'apparition de la Madone adriatique parmi les voisins immédiats de Loreto et le bruit qui entoura cette apparition donnent à penser que sa sphère d'influence régionale s'élargissait. Outre-Atlantique, au Paraguay, deux autres jésuites italiens furent également poussés, par attachement à leur terre natale, à dédier une mission à la madone adriatique. Giuseppe Cataldini (1571-1653) et Simone Mascetti (1577-1658) fondèrent Loreto de Guairá au début du XVII ${ }^{\mathrm{e}}$ siècle; ce fut la première mission jésuite baptisée de ce nom en Amérique du $\mathrm{Sud}^{18}$. Plus au nord,

16. Jonathan Spence, The Memory Palace of Matteo Ricci (New York, Elisabeth Sifton Books, 1984), 259.

17. Ibid., 234. En 1549, la Vierge de Loreto apparut à une citadine de Macerata, Bernardina di Bonino, au cours d'une vision, ce qui eut pour conséquence la construction d'une église dédiée à cette Vierge. Cette église de Macerata fut terminée en 1573, cinq ans avant le départ de Ricci pour l'Inde. Il s'agissait du principal point de repère de la ville. En outre, compte tenu de sa proximité de la basilique de Loreto et de sa taille plus imposante, Macerata servit de lieu d'édition pour la basilique, de manière informelle. Une quantité importante de brochures attestant les guérisons miraculeuses de la Vierge de Loreto furent publiées à Macerata au XVI ${ }^{\mathrm{e}}$ siècle; il est donc probable que Ricci eut accès à quelques-unes de ces brochures quand il vivait à Macerata.

18. Cataldini venait d'Ancona et Mascetti de Castilenti. Ces deux villes étaient près de Loreto, ce qui permet de croire que Cataldini et Mascetti ont d'abord connu la Madone de Loreto en visitant sa basilique 
au Mexique, le jésuite Eusebius Franziskus Kühn (1644-1711), mieux connu aujourd'hui sous le nom espagnol qu'il adopta, Eusebio Kino, récita les litanies de Lorette à répétition avec les matelots au cours de son expédition vers la Californie en 1683, ainsi qu'avec les Amérindiens qu'il rencontra $^{19}$. Kino avait grandi dans le village de Segno, plus de $400 \mathrm{~km}$ au nord de Loreto, dans une région principalement germanophone des Alpes tyroliennes, où la miraculeuse Sainte Maison de Loreto était quelque chose de relativement original. Pourtant, la connaissance qu'avait Kino de la langue italienne et sa passion pour les litanies de Lorette rappelèrent leur terre natale à d'autres jésuites italophones. Dans le territoire de mission de la Nouvelle-Espagne, Kino fut le mentor des nouveaux arrivants, Giovanni Battista Zappa (Juan Bautista Zappa, 1651-1694) et Gianmaria Salvaterra (Juan María Salvatierra, 1648-1717), de Milan. Ce trio de jésuites, célèbre pour la fondation des missions de Californie, formait un genre d'association italienne soudée par la dévotion lorettaine.

Grâce en partie aux Jésuites, Loreto se déplaça et chemina très loin de son berceau italien. En effet, au milieu du XVII ${ }^{\mathrm{e}}$ siècle, le jésuite allemand Wilhelm Gumppenberg écrivit que la Madone de Loreto était l'image de Marie la plus répandue dans le monde ${ }^{20}$. Gumppenberg se fondait sur son expérience personnelle et sur des recherches. Il avait fait un pèlerinage à la basilique de Loreto en 1632. Il avait aussi vécu dans le nord de l'Europe, en Allemagne, à l'époque où cette Madone italienne jouissait d'une grande popularité. Il y ramassa 1200 images différentes de Marie afin de les publier dans son Atlas Marianus, ouvrage qui parut à Ingolstadt entre 1657 et 1659 en quatre volumes relativement minces. La majeure partie des images de Loreto provenaient de la région allemande que Gumppenberg connaissait le mieux ${ }^{21}$.

originale. Voir Antonio Ruiz de Montoya, The Spiritual Conquest Accomplished by the Religious of the Society of Jesus in the Provinces of Paraguay, Paraná, Uruguay and Tape [1639], (St. Louis, Mo, The Institute of Jesuit Sources, 1993), 38-39, 211, n. 39-40, traduction de C. J. McNaspy, s.j.

19. Archivum Romanum Societatis Iesu, Rome (ARSI) Mex. 17, f. 512-524v. (Relación pour 1683 de la deuxième expédition des Jésuites en California); voir aussi Harry W. Crosby, Antigua California: Mission and Colony on the Peninsular Frontier, 1697-1768 (Albuquerque, University of New Mexico Press, 1994), 429, n. 25 .

20. Gumppenberg utilisa même une gravure de la Madone de Loreto assise sur le toit de la Santa Casa pour la page frontispice de son Atlas Marianus (Ingolstadt, 1657-1659). Si l'on se fie à l'appréciation de Gumppenberg, la Vierge de Loreto aurait été légèrement en avance sur l'image romaine de Marie à Sainte Marie Majeure, sur le plan de la diffusion dans le monde. Voir Kirstin Noreen, "The Icon of Santa Maria Maggiore, Rome: An Image and Its Afterlife», Renaissance Studies, 19,5 (2005): 664, n. 11.

21. Frédéric Liechtenberger, Encyclopédie des sciences religieuses (Paris, Librairie Sandoz et Fischbacher, 1888), VII : 311 . 
$\mathrm{Au} \mathrm{XVII}^{\mathrm{e}}$ siècle, des non-Italiens avaient commencé à faire voyager le nom de Loreto ${ }^{22}$. Les ondes toujours plus grandes de la dévotion lorettaine finirent par atteindre l'Amérique française, notamment grâce au jésuite passeur dont nous parlions au début de cette section, le Français Pierre Chaumonot ${ }^{23}$. Avant même de devenir jésuite, alors qu'il était encore un adolescent en fuite, il avait visité la basilique de Loreto, visite qui avait changé sa vie ${ }^{24}$. En route vers Rome, Chaumonot s'était arrêté à ce lieu de pèlerinage; il avait prié la Madone de Loreto de le guérir de ses maux et elle avait exaucé ses prières : un étranger était apparu et avait soigné ses blessures. Chaumonot fit le vœu de se souvenir de la Vierge de Loreto et, plus tard, de construire une chapelle en son honneur.

Le jésuite français Chaumonot s'exprimait avec une clarté exceptionnelle sur ce que le nom et le lieu de Loreto signifiaient pour lui. La Sainte Maison de Loreto en était l'incarnation: humble demeure de Marie qu'il entreprendrait plus tard de reproduire, selon les dimensions exactes, dans la marge pionnière canadienne. Nom et structure physique étaient intrinsèquement liés dans l'esprit de Chaumonot, non seulement dans le cas de Loreto. Le jésuite amalgamait souvent nom et architecture sous cet angle particulier: un nouveau sens se greffait littéralement sur l'ancien, sans l'effacer. Lorsqu'il était attribué à l'étranger, Loreto portait par conséquent une partie du poids du Loreto original, celui d'Italie, mais une dimension toute neuve, active, s'ajoutait aussi. Pour Chaumonot et pour plusieurs de ses contemporains, Jésuites et autres, les noms annonçaient à la fois le changement et la continuité de manière aussi tangible qu'une nouvelle

22. En outre, des religieux non jésuites ont transporté la dévotion de l'Italie vers le monde atlantique français, notamment l'ordre catholique des Sulpiciens. Jean-Jacques Olier, fondateur de l'ordre, fut guéri d'une affection aux yeux à Loreto, en 1630, et plus tard rendit public son attachement à cet endroit. Près de Paris, au séminaire de Saint-Sulpice d'Issy-les-Moulineaux, il y a une chapelle construite sur le modèle de la Sainte Maison à la fin des années 1690. Un des supérieurs français des Sulpiciens au Canada, François Vachon de Belmont (1645-1732), fit construire en 1697 un fort qu'il appela «Lorette », près de Montréal, au Sault-au-Récollet, afin de protéger la jeune mission sulpicienne de La Montagne. Bibliothèque Nationale de France, Paris (BnF), Ms. FR 11758, Plan de l'église de la Vierge, 1650-1690); voir aussi JeanJoseph Expilly, Dictionnaire géographique, historique et politique des Gaules et de la France (Paris, Desaint et Saillant, 1764), III : 869.

23. Chaumonot était en bonne compagnie parmi les Français qui répandaient également la dévotion à la Madone de Loreto. Il était un contemporain de Jean-Jacques Olier (voir note précédente). En outre, à l'époque de Chaumonot, en 1646, l'archevêque de Paris, Gondi, approuva la création d'une confrérie vouée à Notre-Dame-de-Lorette. Voir Henri Gourdon de Genouillac, Paris à travers les siècles : Histoire nationale de Paris et des Parisiens... (Paris, F. Roy, 1890), II : 360.

24. Pour l'article le plus récent sur Chaumonot et ses voyages, voir Allan Greer, «Un jésuite errant en Europe et en Amérique: le père Chaumonot", dans La Nouvelle-France et le monde (Montréal, Boréal, 2009), 149-170. 
église. Le mot et la structure pouvaient l'un comme l'autre servir de preuve d'une nouvelle ère stimulante de dynamisme catholique.

Regardons, par exemple, ce que fit Chaumonot la soirée précédant son départ pour le Nouveau Monde. Sur le point d'entreprendre un voyage dont il était possible qu'il ne revienne jamais, Chaumonot ajouta des couches de vernis religieux à son nom. Comme il l'explique dans son autobiographie, il choisit d'adopter deux nouveaux noms: Pierre Chaumonot devint PierreJoseph-Marie. Il décrit ce moment d'addition significatif et créatif comme étant en partie inspiré par les ruines antiques de la ville de Rome. Chaumonot écrit:

Il y a dans Rome une chapelle de St. Joseph, où de temps en temps s'assemble la Confrérie qui lui est dévouée. Je visitai ce saint lieu quelques jours après que j'eus pris les noms de Joseph-Marie et je sus que cette dévote chapelle étoit batie sur la prison où St. Pierre avoit été enfermé et chargé de chaines. Il me vint alors en pensée que comme on avoit bati une chapelle à St. Joseph sur un lieu qui étoit honoré du nom du prince des apôtres, de même Dieu pouvoit bien se servir de moi qui avois reçu le nom de Pierre à mon baptême pour faire une chapelle où le digne nourricier de Jésus fut honoré avec sa divine épouse. Il me sembla même qu'au sujet de la future Lorette du Canada, Notre Seigneur me feroit la grace d'avoir au moins une petite part à cette grande promesse qui fait la gloire de mon saint patron: «Tu es Petrus et super hanc petram ædificabo ecclesiam meam. $»^{25}$.

La vision profondément spirituelle du monde qu’avait Chaumonot était enveloppée dans des noms et dans les lieux anciens et familiers qu'ils désignaient. Comme il l'expliquait, il garda «Pierre» pour ses liens avec le fondateur de l'Église, saint Pierre. Dans un élan d'enthousiasme, il ajouta aussi «Joseph» et «Marie » à son nom de baptême afin de pouvoir porter avec lui ces deux figures spirituelles comme fondements de ses nouvelles églises ${ }^{26}$. Il empilait des noms sur sa personne, faisant délibérément écho à l'architecture de Rome.

Comme l'a remarqué l'historien Allan Greer, Chaumonot était obsédé par les noms. Il jonglait si adroitement avec eux, au fur et à mesure qu'il

25. Chaumonot décrit ce moment dans son autobiographie, La Vie du R.P. Pierre Joseph Marie Chaumonot de la Compagnie de Jésus, missionnaire dans la Nouvelle France, écrite par lui-même par ordre de son supérieur, l'an 1688 (Nouvelle York, Isle de Manate, à la Presse Cramoisy de Jean-Marie Shea, 1858), 48-49.

26. «Environ dans le même tems je sus que le Canada avoit pour patron le glorieux St. Joseph: ce qui excita ma dévotion à demander à notre Révérend Père Général qu’il me permit de me nommer Joseph Marie: ce qu'il m'accorda sur les raisons que je lui en apportai, de me mettre sous la puissante protection de ces deux époux vierges, de reconnoitre par là les obligations que je leur ai et de m'exciter à l'imitation de leurs vertus. » Ibid., 48. 
entrait dans de nouveaux environnements, qu'il est clair qu'ils signifiaient davantage à ses yeux qu'une simple traduction. Chaque nouvelle variante servait à célébrer un ajout à sa liste toujours croissante d' "alliances et [d'] affiliations" personnelles ${ }^{27}$. Chaumonot était entré dans la vie en répondant au nom que lui avaient donné ses parents par l'entremise de l'Église catholique lors de son baptême, en France: Pierre Chaumonot. Il était encore jeune lorsqu'il passa à une version italienne de ce nom: Pietro Calmonotto. Il s'était enfui vers l'Italie et avait employé ce nom italianisé pour s'y établir. Dans ce pays, en outre, ses confrères jésuites le désignaient par son nom latin: Petrus Calmonottus. Il complexifia encore les façons possibles de s'adresser à lui en adjoignant «Joseph-Marie» à son prénom au cours de sa formation de jésuite, comme nous l'avons vu plus haut ${ }^{28}$.

Ces changements de nom, chez Chaumonot, ont été étudiés comme une stratégie d'adaptation profondément personnelle et individuelle, comme la recherche d'un chez-soi, d'une famille et d'une mère ${ }^{29}$. Mais Chaumonot n'était pas le seul à changer ainsi de nom. Au XVII ${ }^{e}$ siècle, il était assez normal pour les Jésuites d'accumuler une longue liste de surnoms au cours de leur carrière ${ }^{30}$. Bien que la décision de modifier leur nom ne fût pas prise à la légère par les Jésuites, ceux-ci répétaient l'expérience au cours de leur vie pour annoncer les transitions, qu'elles soient banales ou spectaculaires. Ici, j'aimerais signaler qu'il arrivait souvent que l'on ajoute des noms, mais rarement qu'on en retire. Par exemple, Pierre ne perdit pas son nom de baptême ni son nom de famille, Chaumonot, lorsqu'il entra dans la Compagnie de Jésus. Il gagna plutôt les noms de «Joseph» et de «Marie». Les nouveaux noms n’effacèrent pas le passé de Chaumonot. Les changements prirent la forme de couches de noms appliquées soigneusement les unes après les autres.

27. A. Greer, «Un jésuite errant...», op. cit., 170.

28. Il arrivait fréquemment que Chaumonot mêle tous ces noms lorsqu'il signait sa correspondance, tout en favorisant l'italien, peut-être parce que cette langue lui rappelait l'endroit où il avait vécu l'essentiel de ses années de formation. Voir, par exemple, une lettre qu'il écrivit du Canada à Filippo Nappi, en 1641, et qu'il signa "Giuseppo Maria Calmonetti», ARSI Gall. 109. Historia Missionis Canaden, 1611-1659, I: f. $112 \mathrm{v}$.

29. A. Greer, «Un jésuite errant...», op. cit., 149-170.

30. Cette stratégie semble particulièrement typique des jésuites qui ont travaillé dans le monde atlantique français, bien que les recherches d'Allan Greer donnent à penser que ces changements de nom ont peut-être été communs aux missionnaires jésuites partout à l'étranger, en raison de leur travail interculturel qui nécessitait la maîtrise de nouvelles langues. Greer fournit un autre exemple français de cet archétype du jésuite adaptable et du "polyglotte changeant» en la personne d'un contemporain et collègue de Chaumonot au Canada, le jésuite Claude Chauchetière, que l'on voit à l'œuvre dans Catherine Tekakwitha et les jésuites : la rencontre de deux mondes (Montréal, Boréal, 2007), traduction Hélène Paré. 
Il est significatif, et tout à fait à l'opposé de l'idée de noms nouvellement imposés destinés à remplacer définitivement les anciens, que les Jésuites ne semblent pas s'être inquiétés de la multiplicité des noms. Il s'agissait d'un modèle de superposition qu'ils connaissaient bien en Europe, où le paysage lui-même était un palimpseste de noms que l'on avait grattés pour en graver de nouveaux. Comme nous l'avons vu, Chaumonot réunissait l'architecture et les noms dans l'extrait de son autobiographie cité plus haut, où il s'émerveillait devant les couches de Rome, ville où l'ancien et le nouveau étaient si étroitement liés l'un à l'autre qu'ils semblaient être interdépendants. Un grand nombre d'histoires écrites par des religieux et des spécialistes de l'Antiquité venaient renforcer son expérience personnelle. Ainsi que les bâtiments, les noms se plaçaient dans les reconstructions du passé de ces érudits comme des indices de la provenance des gens et de leur manière de se rendre dans un lieu. Jean Bodin (1530-1596), philosophe politique et juriste français, était un écrivain de renom qui attira l'attention sur l'étymologie dans son traité de 1566, Methodus ad facilem historiarum cognitionem (ou La Méthode pour étudier l'Histoire) ${ }^{31}$.

Arrêtons-nous à un exemple dans lequel Bodin employa un nom pour élucider des origines passées. Il décrivit la migration des peuples wallons, appelés Ouallones en latin. Ces francophones avaient erré jusqu’en Belgique à une certaine époque. Bodin confronta leur langue et leur nom et élabora une théorie voulant que ce groupe ait reçu son étiquette en se demandant les uns aux autres «Où allons-nous?», lorsqu'ils parcouraient le territoire belge ${ }^{32}$. Le raisonnement de Bodin n'était pas si tiré par les cheveux lorsqu'on le comparait à la véritable confusion des désignations qui régnait outre-Atlantique, à son époque. Antonio de Ciudad Real décrivit comment les Espagnols décidèrent du nom à donner à la péninsule du Yucatan, au Mexique, en 1588. Ciudad Real écrivit: «Lorsque les Espagnols découvrirent ce territoire, leur chef demanda aux Indiens quel nom ils lui donnaient; comme ils ne le comprenaient pas, ils dirent uic athan, ce qui signifie que dites-vous ou que parlez-vous, que nous ne vous comprenons pas. Puis l'Espagnol donna l'ordre d'inscrire qu'on l'appellerait Yucatan ${ }^{33}$.»

31. Sur le contexte historique entourant Bodin et ses contemporains, voir Donald R. Kelley, Versions of History from Antiquity to the Enlightenment (New Haven, CT, Yale University Press, 1991), 1-17, 380-394.

32. Anthony Grafton, What Was History? The Art of History in Early Modern Europe (New York, Cambridge University Press, 2007), 166-167.

33. Antonio de Ciudad Real, cité dans Inga Clendinnen, Ambivalent Conquests: Maya and Spaniard in the Yucatan, 1517-1570 (New York, Cambridge University Press, 2003), x. Il existe des explications semblables concernant l'attribution du nom «Pérou» par les Espagnols. Voir Paul Firbas, «Narration and 
Les noms peuvent receler leur propre étymologie : ceux que des Jésuites comme Chaumonot ont apportés ou attribués dans les Amériques témoignent de cette possibilité. Chaumonot fit des choix personnels de noms qui l'aidèrent à donner un sens à sa vie et qui ouvrirent la voie aux futurs chercheurs qui, à l'instar de Bodin, se pencheraient sur les traces de son passage. Il avait confiance que les autres Jésuites, les catholiques et les habitants des régions américaines limitrophes liraient sa collection d'appellations étrangères et verraient ainsi les chemins reliant l'ancien et le nouveau.

L'hypothèse de Chaumonot s'affermit devant les pratiques d'attribution de nom qu'il rencontra en Amérique du Nord. Au Canada, les Hurons lui donnèrent deux nouveaux noms: «Aronhiatiri» et «Héchon». Le fait que Chaumonot soit devenu «Héchon» est significatif, tant par le choix des Hurons de lui attribuer un nom recyclé, que par le choix de Chaumonot d'embrasser cette désignation huronne. Lorsque Chaumonot était arrivé au Canada, les Hurons l'avaient d'abord appelé "Aronhiatiri», ce qui signifie «celui qui porte le ciel $^{34}$ ». Il est probable que ce nom faisait allusion à la profession de Chaumonot, missionnaire qui apportait le ciel aux Hurons. Après plusieurs années parmi les Hurons, cependant, ceux-ci le surnommèrent cérémonieusement "Héchon" après le martyre de son collègue, le jésuite Jean de Brébeuf. Les Hurons avaient appelé Brébeuf "Héchon», qui était peut-être une tentative de prononcer son prénom, «Jean», dans la langue wendat. Ce nom fut associé tellement étroitement aux compétences uniques de Brébeuf qu’il finit par revêtir le sens d' "étranger doué en langue huronne ${ }^{35}$ ".

Chaumonot et Brébeuf étaient tous deux reconnus pour leurs compétences linguistiques et c'est peut-être ce qui explique que Chaumonot fut invité à partager le nom du missionnaire jésuite décédé, son prédécesseur. Les Hurons croyaient que l'adoption du nom d'une personne décédée permettait à son esprit de continuer à vivre dans une nouvelle personne ${ }^{36}$. Il y a d'intéressantes convergences entre ces pratiques autochtones et les pratiques européennes de réappropriation et de superposition de noms. À Florence, au XV siècle, par exemple, les familles transmettaient souvent

Etymologies: Colonial Writing and the Name "Peru"”, communication présentée dans le cadre du Program in Latin American Studies, Princeton University, 3 avril 2001.

34. John Steckley, Words of the Huron (Waterloo, Wilfrid Laurier University Press, 2007), 238.

35. Ibid., 236.

36. A. Greer, «Un jésuite errant...», op. cit., 160; sur une tradition semblable chez les Iroquois, voir John Demos, The Unredeemed Captive: A Family Story from Early America (New York, Vintage Books, 1995), 141. 
aux enfants le nom de parents décédés, comme pour les réincarner ${ }^{37}$. Ainsi, l'idée d'hériter d'un nom de cette manière peut avoir été familière à Chaumonot. Il est certain qu'il n'abordait pas cette coutume huronne comme s'il s'agissait d'une curiosité étrangère.

Mais Chaumonot ne se contentait pas de tolérer le nom réattribué. Il acceptait la nouvelle identité de "Héchon" aussi entièrement qu’il avait adopté la série des autres noms qu'il avait lui-même choisis, quand il était en Europe. John Demos note un cas semblable de colons en Amérique du Nord qui adoptèrent de bon cœur les nouveaux noms qui leur furent donnés, lorsqu'il raconte l'histoire d'une captive anglaise, Eunice Williams, emmenée du Massachusetts jusque chez les Iroquois de Kahnawake (Canada) au début du XVIII ${ }^{e}$ siècle et qui choisit d'y rester plutôt que de retourner chez elle. À l'arrivée d'Eunice à Kahnawake, elle reçut un nom iroquois (A’ongote). Des années plus tard, un nom français (Marguerite) $s^{\prime} y$ ajouta lors de son baptême dans la religion catholique ${ }^{38}$. Elle fut à l'aise d'utiliser tantôt A'ongote, tantôt Marguerite pour le reste de sa vie à Kahnawake, tout comme Chaumonot choisit de répondre aux noms de Héchon et de Pierre-Joseph-Marie jusqu’à la fin de ses jours. La superposition de noms était une source de fierté, car elle indiquait simultanément et l'intégration et le souvenir.

\section{DES NOMS EN LITIGE: LES HURONS DE LA JEUNE-LORETTE}

Les noms de lieux devenaient-ils, comme les noms de personnes, des couches supplémentaires soigneusement choisies et conférées pour indiquer l'origine et les attaches des gens? Si c'est le cas, cette réalité vécue contredit l'interprétation stéréotypée selon laquelle les noms européens et catholiques importés en Amérique du Nord n'indiquent que l'intransigeance, la conquête ou la possession. Elle donne plutôt à penser qu'un nom de lieu étranger pouvait, comme le nom personnel «Héchon» pour Chaumonot, dénoter une connotation positive. Ayant cela à l'esprit, tournons-nous enfin vers un exemple d'attribution d'un nom dans le Nouveau Monde, celui que Chaumonot donna à une mission huronne en s'inspirant de la Vierge de Loreto. Je commencerai par exposer brièvement la façon dont Chaumonot choisit de conférer le nom de Loreto à cette communauté particulière. Mais je terminerai en attirant l'attention sur la façon dont ce nom fut revendiqué et contesté par les Hurons eux-mêmes.

37. Edward Muir, Ritual in Early Modern Europe (New York, Cambridge University Press, 2005), 25.

38. J. Demos, The Unredeemed Captive..., op. cit., 141-142, 151-154. 
Vers la fin de sa carrière de missionnaire, en 1674, Chaumonot réalisa enfin son vœu et construisit au Canada une réplique de la Sainte Maison de Loreto. Il la situa chez un groupe exceptionnel de néophytes hurons pour lesquels il éprouvait une grande loyauté. Ces convertis hurons avaient déménagé cinq fois depuis que Chaumonot avait fait leur connaissance. À chaque déménagement, il fallait quitter l'endroit qu'ils avaient occupé pendant au moins un an afin d'établir une nouvelle communauté, à plusieurs kilomètres du lieu précédent. Pensons au bouleversement que cela entraînait pour les membres les plus âgés de la mission de Lorette. La personne huronne qui s'était établie dans la première mission SainteMarie, au bord de la baie Georgienne, dans les années 1640, avait ensuite subi les transplantations suivantes: à Sainte-Marie II sur l'île Gahoendoe, à Québec, à Sainte-Marie III sur l'île d'Orléans, de nouveau à Québec, puis à Notre-Dame-de-Foye. Si elle avait dans la jeune vingtaine au moment de son déménagement à Sainte-Marie I, elle aurait eu dans la cinquantaine au moment du transfert à Notre-Dame-de-Lorette. Bien que plusieurs des chefs de la communauté huronne de Québec fussent des réfugiés plus jeunes, non originaires de la baie Georgienne, il y avait à Notre-Damede-Lorette un noyau de plus de 30 aînés qui avaient vécu tous ces déplacements ${ }^{39}$.

Au cours de ces cinq relocalisations, ce petit groupe de Hurons qui choisit de lier son sort aux Jésuites porta successivement à peu près autant de noms ${ }^{40}$. Il avaient d'abord été désigné, en 1639, comme la mission jésuite de Sainte-Marie-des-Hurons, désignation que ses membres réussirent à garder sous diverses formes (Sainte-Marie I, Sainte-Marie II) lorsque la guerre les eut chassés de leur établissement d'origine dans la baie Georgienne. Séparés et réfugiés près de Québec, les survivants se rassemblèrent sous le nouveau parapluie de «Notre-Dame-de-Foye» en 1667. Chaumonot les resitua dans un autre village de mission en 1672, baptisant celui-ci «Notre-Dame-de-Lorette», en l'honneur de la réplique de la Sainte Maison lorettaine qu'il plaça en son centre ${ }^{41}$. Pour Chaumonot, l'événe-

39. Bruce Trigger, The Children of Aataentsic: A History of the Huron People to 1660 (Montréal, McGillQueen's University Press, 2000), 763, 767, 820-825.

40. Des centaines de Hurons choisirent une autre direction et abandonnèrent la mission des Jésuites. Ibid., 750. Voir également Daniel K. Richter, «Iroquois vs. Iroquois : Jesuit Missions and Christianity in Village Politics, 1642-1686», Ethnohistory 32,1 (1985): 1-16.

41. Félix Martin, s.j., "Précis historique sur la mission Huronne et sur les migrations de ce peuple jusqu’à nos jours", dans F. J. Bressani, s.j., Relation abrégée de quelques missions des Pères de la Compagnie de Jésus dans la Nouvelle France [Macerata, 1653]. Traduit de l'Italien et augmenté d'un avant-propos, de la biographie de l'auteur, et d'un grand nombre de notes et de gravures, par le R. P. F. Martin, S.J. (Montréal, Presses à Vapeur de John Lovell, 1852). 
ment semble avoir été un exemple de plus de superposition spirituelle significative - comme lorsqu'il avait ajouté «Joseph» et "Marie» à son prénom. Il voulait que ces Hurons jouissent de la grâce et de la protection additionnelles de la Madone de Loreto.

Quel sens cela eut-il pour les Hurons, cependant? En écrivant au sujet d'une mission canadienne de la même époque qui porta plusieurs noms - Kahnawake -, l'historien John Demos observe que "plusieurs appellations [d'établissements autochtones du XVII ${ }^{\mathrm{e}}$ siècle] reflètent directement l'ambiguiité de leur situation culturelle et géographique - et l'extraordinaire complexité de leur histoire ${ }^{42}$ ». L'établissement iroquois dont il est question, par exemple, revêtit successivement au moins quatre noms correspondant à des déplacements sur de courtes distances ${ }^{43}$. Ce fut d'abord un village iroquois appelé Kentake que les Français surnommèrent «La Prairie». Il fut renommé «St. François-Xavier-des-Prés» lorsque le père Raffeix, missionnaire jésuite, y déménagea sept familles iroquoises catholiques. La mission adopta le nom "Kahnawake» («lieu des rapides») en 1676 , lorsqu'elle déménagea tout près, au «Sault St. Louis», comme les Français appelaient le rivage longeant les rapides du Saint-Laurent. SaultSaint-Louis / Kahnawake fut reconnu par les Français et les Iroquois, qui utilisèrent les deux appellations, la française et l'iroquoise ${ }^{44}$.

Contrairement à Sault-Saint-Louis/Kahnawake, toutefois, la mission de «Lorette» devint connue presque exclusivement par son nom francoitalien, «Lorette». On peut le constater tout autant dans les chroniques françaises du XVII ${ }^{\mathrm{e}}$ siècle que dans les «autohistoires» des descendants de la collectivité huronne ${ }^{45}$. Cette forte identification des Hurons avec «Lorette» s'est produite en partie à cause de la concurrence locale autour du nom et du site. Aux yeux des colons français de Québec, c’était une nouveauté qui tenait presque du miracle de voir une Sainte Maison de Lorette apparaître tout d'un coup à une distance assez courte, à pied, de leur ville ${ }^{46}$. En fait, on pouvait voir la ville fortifiée depuis la réplique de

42. J. Demos, The Unredeemed captive..., op. cit., 120.

43. Louis Lavallée, La Prairie en Nouvelle-France, 1647-1760. Étude d'histoire sociale (Montréal, McGillQueen's University Press, 1992), 74.

44. Voir A. Greer, «Kahnawake: une collectivité iroquoise et chrétienne», chap. 4 de Catherine Tekakwitha et les jésuites, op. cit.

45. Georges Sioui, lui-même d'origine huronne-wendate, souligne que le principal accès à ce sentiment d'appartenance à un lieu passe par les sources françaises, mais il reconnaît aussi l'identification des autochtones avec «Lorette». Voir Georges E. Sioui, Pour une autohistoire amérindienne. Essai sur les fondements d'une morale sociale (Québec, Presses de l'Université Laval, 1989).

46. Archives de la Société de Jésus, province du Canada français, Saint-Jérôme (ASJCF), Doc. n 324 (1675 Relation du Père Martin Bouvart, S.J.), f. 7-8. 
la Sainte Maison de Lorette. Québec se trouvait à quelque huit kilomètres en suivant la vallée de la rivière Saint-Charles vers l'est. Au cours de ses premières années, la réplique de la Sainte Maison introduite par Chaumonot attira un afflux régulier de pèlerins français de Québec. Trois des premiers miracles rapportés concernaient des résidants de Québec ${ }^{47}$.

L'attention de ses voisins de la ville eut des conséquences pour la petite communauté huronne de Lorette qui comptait environ 150 personnes ${ }^{48}$. Un des récits portant sur le culte rendu dans ce bâtiment apporte des précisions:

Comme aux jours de fête, nos Sauvages ne pourraient pas être commodement introduits dans ce petit sanctuaire, à cause des Français qui viennent en grand nombre faire leurs devotions, et qui n'entrent aussi dans ce saint lieu qu'après leur communion, ils ont pris pour eux les jours ouvriers; ainsi il y a tous les jours une famille qui se confesse et qui communie pour ce sujet; et lorsque le tour des cabanes est achevé, ils recommencent avec autant et plus de ferveur qu'à la première fois ${ }^{49}$.

Il faut remarquer qu'outre la participation à la communion, les Jésuites observent que, tout au long des années 1670, les Hurons viennent prier à la chapelle aux premières lueurs du jour ${ }^{50}$. Les Jésuites étaient portés à

47. Un donateur français fut guéri d'une maladie, Marie Pelletier, épouse de Jean Denys, marchand français, fut guérie et un garçon français, du nom de Losier, fut guéri d'un problème respiratoire. Voir ASJCF, Fonds Joseph Chaumonot, BO-258, Cahier 2, doc. 8: Martin Bouvart au R. P. Claude Boucher, "Avantages de la Dévotion de Lorette confirmée par les faveurs obtenues de Dieu pendant l'année 1674 ».

48. Selon un recensement de 1685 , la maladie avait réduit la population de la mission de son nombre maximum, soit 300 habitants, une décennie plus tôt, à seulement 146. Cette donnée impressionne encore plus quand on la compare à la population des autres missions jésuites en 1685 : Saint-François-de-Sales comptait 680 Iroquois, Saint-Louis ou Saint-François-Xavier-du-Sault, 600 Abénaquis, et Saint-François et Bécancour, environ 500 Abénaquis. Voir Camille de Rochemonteix, s.j., Les Jésuites et la Nouvelle-France au XVIII Siècle d'après beaucoup de documents inédits(Paris, Letouzey et Ané, Éditeurs, 1896), III : 375-378, en particulier page 376. En outre, la population ne s'est pas rétablie au XVIII ${ }^{\mathrm{e}}$ siècle. En 1752, lorsque le colonel Louis Franquet visite la Jeune-Lorette, il remarque que le village ne comprend que 25 familles logeant dans seulement 17 maisons, soit 120 habitants au total. Voir "Voyage de Québec au village de Lorette sauvage, fait à Québec le 28 décembre 1752», dans [Franquet], Voyages et mémoires sur le Canada (Montréal, Éditions Élysée, 1974 [1889]), 107, ou Bibliothèque et Archives Canada, (BAC), Collection Louis Franquet (1751-1753), Récits des voyages et mémoires sur le Canada, 1752-1753, II : 144.

49. Dans «Mission des Hurons... 1673-1674», Félix Martin, s.j., Relations inédites de la Nouvelle-France (1672-1679) pour faire suite aux anciennes relations (1615-1672) avec deux cartes géographiques (Paris, Charles Douniol, 1861), I: 318.

50. Jeanne Asseuragenhaon, par exemple, fut l'une des nombreuses dévotes de l'aube à Lorette. BAC, Collection Félix Martin (1611-1776), MG18-H27, «De la Mission des Hurons... », f. 99-100. Sur la communion: les missionnaires jésuites réglementaient l'accès à cette cérémonie afin de promouvoir la pratique catholique de la confession. Ils tenaient également un compte précis des communiants comme mesure des conversions réussies. Pour une intéressante étude de cas - où la comparaison avec les Hurons de Québec est possible -, voir les façons des jésuites d'aborder la communion et les réactions des indigènes, dans le Mexique du XVIII ${ }^{\mathrm{e}}$ siècle, décrits par William L. Merrill, "Conversion and Colonialism 
interpréter et à louanger ce culte matinal comme une manifestation de piété. Étant donné les foules des jours de fête, en particulier, il est possible qu'un des motifs de ces visites à l'aube ait été que c'était le moment de la journée où les Hurons, qui habitaient à deux pas de la chapelle, n'avaient pas à jouer des coudes pour entrer dans la petite maison.

En outre, même si en 1687 le jésuite Chaumonot rapportait que l'ordre régnait à la mission, son collègue Michel-Germain Decouvert se désolait de l'influence que les pèlerins de Québec exerçaient sur les Hurons. Avec les pèlerins venaient les vendeurs d'eau de vie. «Le village est maintenant fort détraqué, souillé d'ivrognerie et d'impureté», rapporte Decouvert à la fin des années $1680^{51}$. À Lorette, Michel-Germain Decouvert avait assisté Chaumonot et un autre jésuite d'expérience, Julien Garnier. Jésuite originaire de Normandie, Decouvert avait été envoyé au Canada alors qu’il n'était plus tout jeune et il avait 42 ans lorsqu'il fut nommé supérieur de Lorette en $1695^{52}$. Il se montra plein de confiance et de dynamisme dans ses projets pour aider les Hurons. Après une campagne axée sur l'élimination de l'alcool à Lorette, il décida qu'il était dans le meilleur intérêt des catholiques hurons de se reloger loin de la dépravation de Québec ${ }^{53}$.

C’est ainsi que, deux décennies seulement après que Chaumonot eut donné à la mission huronne le nom de «Lorette», les Hurons furent déplacés de nouveau vers un endroit qui les éloignait encore de quelques kilomètres de Québec ${ }^{54}$. Cette fois, les Hurons n'adoptèrent pas un nouveau nom pour désigner leur mission: ils s'accrochèrent à leur vieux nom. Ils appelèrent le lieu de leur nouvelle mission "Jeune-Lorette» pour la distinguer de l'ancien site de la réplique, qui fut renommé «Ancienne-Lorette » pour plus de clarté. Si les Jésuites ont peut-être encouragé le maintien du lien de cette communauté avec le nom «Lorette», les Hurons eux-mêmes

in Northern Mexico: The Tarahumara Response to the Jesuit Mission Program, 1601-1767», dans Robert W. Hefner, dir., Conversion to Christianity: Historical and Anthropological Perspectives on a Great Transformation (Berkeley, University of California Press, 1993), 144-147.

51. Michel-Germain Decouvert, cité par Camille de Rochemonteix, s.j., Les Jésuites et la Nouvelle-France au XVIII siècle d'après beaucoup de documents inédits (Paris, Letouzey et Ané, Éditeurs, 1896), III: 392-394.

52. Charlotte Gros-Louis et Céline Gros-Louis, La Chapelle huronne de Lorette, 1730-1980 (Village-desHurons, Charlotte Saint-Louis et Céline Saint-Louis, 1980), 105. Decouvert vécut les deux décennies suivantes dans cette mission; il mourut à la Jeune-Lorette à l'âge de 62 ans.

53. Apparemment, les efforts de Decouvert pour éradiquer l'alcoolisme dans la paroisse huronneiroquoise eurent assez d'effets positifs pour que l'un de ses collègues, Louis D'Avaugour, le salue comme le "sauveur de la mission [de Lorette]» en 1710. M.-G. De Rochemonteix, Les Jésuites et la NouvelleFrance..., op. cit., 392-394.

54. Léon Gérin, La Seigneurie de Sillery et les Hurons de Lorette (Ottawa, Hope \& Son, 1901). Voir, également, la collection d'interprétations des documents juridiques portant sur Lorette dans Denis Vaugeois, dir., Les Hurons de Lorette (Sillery, Éditions du Septentrion, 2011). 
semblent être restés fidèles à cette appellation catholique plutôt que d'opter pour une solution de rechange autochtone et évidente: "Kabir Kouba». Le site du nouveau village, la Jeune-Lorette, surplombait la chute spectaculaire de Kabir Kouba. Au cours de leurs premières années à cet endroit, les Hurons relocalisés trouvèrent deux cavités dans la pierre à la base de cette chute déjà sacrée et décrivirent ces cavités comme étant les empreintes de la Vierge Marie. Ils imaginaient la sainte mère catholique présente dans les grottes et les eaux rugissantes au bas de Kabir Kouba, là où elle avait combattu et vaincu le diable qui avait la forme d'un serpent ${ }^{55}$. Au lieu d'étendre le nom de ce point de repère physique et spirituel, "Kabir Kouba », à leur mission transplantée, les Hurons continuèrent de différencier les désignations et les sites étroitement juxtaposés. En effet, Kabir Kouba semble avoir connu un regain de sens religieux, avec l'établissement de la Jeune-Lorette au début des années 1700, plutôt que l'inverse.

Les Hurons n'abandonnèrent pas leur nom d'adoption, non plus qu'ils cédèrent facilement leur réplique de la Sainte Maison. Le jésuite Decouvert lui-même proposa aux Hurons de démanteler la réplique héritée de Chaumonot et de la reconstruire à leur nouvelle mission. Sous sa direction, les Hurons commencèrent à démonter toute la décoration intérieure de la petite chapelle et à transporter ces objets ainsi que la plupart des statues de Marie jusqu'à la Jeune-Lorette, quelques kilomètres plus loin ${ }^{56}$. Les colons français étaient furieux. À cette époque, certains d'entre eux habitaient assez près de l'Ancienne-Lorette pour fréquenter la Sainte Maison lors de la messe du dimanche. Ils firent appel à l'évêque de Québec, $\mathrm{M}^{\mathrm{gr}} \mathrm{de}$ Saint-Vallier (1653-1727), pour lui demander d'intervenir ${ }^{57}$.

$\mathrm{M}^{\mathrm{gr}}$ de Saint-Vallier proposa un compromis original qui eut pour effet de faire durer le conflit entre la Jeune et l'Ancienne-Lorette. En 1698, il décida que les colons français pourraient garder la réplique de la Sainte Maison bâtie sous les ordres de Chaumonot à condition qu'ils dédommagent les Hurons de la Jeune-Lorette en accomplissant une corvée pour aider les Hurons à construire une chapelle qui remplacerait la réplique.

55. C. Gros-Louis et C. Gros-Louis, La Chapelle huronne de Lorette..., op. cit., 93, 95. En outre: visite guidée de la chute Kabir Kouba et de l'église Notre-Dame-de-Lorette, organisée par la Maison Arouanne, Conseil de la Nation Huronne-Wendat, Wendake (Québec), juin 2006.

56. La majeure partie des dons offerts à la chapelle de la Sainte Maison de Chaumonot se sont retrouvés chez les Hurons à la Jeune-Lorette, y compris le reliquaire d'argent de Chartres. C. Gros-Louis et C. Gros-Louis, La Chapelle huronne de Lorette..., op. cit., 212.

57. Ibid. Jean-Baptiste de La Croix de Chevrières de Saint-Vallier fut le deuxième évêque de Québec; il arriva de France pour reprendre le poste de $\mathrm{M}^{\mathrm{gr}}$ de Laval, auquel il succéda officiellement en 1688. 
L'évêque alloua une somme d'argent à chaque partie afin de contribuer à la construction et les laissa définir les détails ${ }^{58}$. Ainsi, 40 colons français se présentèrent à la Jeune-Lorette pour y travailler pendant trois jours. Ensuite, le curé affecté à l'Ancienne-Lorette, le père Bouvard, fit allègrement rapport à l'évêque que les Français avaient accompli leur devoir de travail. Le jésuite Decouvert manifesta son désaccord avec véhémence. Tout ce que les Français avaient fait était de couper du bois, écrivit-il en colère; dès qu'ils voudraient vraiment s'acquitter de leur partie du marché, il faudrait qu'ils reviennent. Le plaidoyer de Decouvert ne reçut aucune réponse. Ce n'est qu'en 1710 que les Hurons de Jeune-Lorette terminèrent la construction de leur nouvelle chapelle $e^{59}$.

Mais la rupture ne finit pas là. Les descendants du groupe original de Hurons transmettent encore une histoire à propos du voyage mystérieux de deux objets en particulier entre l'Ancienne et la Jeune-Lorette ${ }^{60}$. Le premier était une statue de la Madone de Loreto. On disait que les Hurons avaient emporté la statue de la chapelle de la Sainte Maison de l'AncienneLorette jusqu'à leur nouvelle chapelle, à la Jeune-Lorette. Au cours de la nuit, cependant, la statue revint dans le village français de l'AncienneLorette. Les Hurons s'en emparèrent de nouveau, mais de nouveau la statue retourna là d'où elle venait. À peu près à la même époque, un basrelief en bois représentant la Sainte Maison de Loreto aurait disparu de l'Ancienne-Lorette pour réapparaître comme par magie à la JeuneLorette, où elle demeura. Cette histoire orale à propos des allers-retours des reliques a été interprétée comme correspondant à une autre intervention de l'évêque de Québec. Étant donné les rivalités à propos des objets de piété de la première Lorette, l'évêque a peut-être joué de nouveau les arbitres afin d'encourager le partage. La tradition catholique huronne reflète cette décision selon laquelle les Français de l'Ancienne-Lorette pouvaient garder la stature de la Madone de Loreto, alors que les Hurons de la Jeune-Lorette pouvaient emporter avec eux la sculpture en bois de la Sainte Maison ${ }^{61}$.

58. Ibid., 18.

59. Ibid. La nouvelle chapelle ne devait pas être une reproduction de la réplique de Chaumonot ni de la Sainte Maison de Loreto, bien que les dimensions fussent semblables.

60. Il ne s'agit pas des seuls mystères à survenir pour résoudre des conflits locaux, selon la tradition catholique huronne. En juillet 1730, lorsque les Hurons étaient à construire une deuxième chapelle pour remplacer la première, ils se disputèrent à propos du lieu où elle devait s'élever. Après la querelle, il se mit à neiger - en juillet. Les Hurons construisirent l'église là où la neige était tombée, voyant en celle-ci un signe de la Vierge Marie indiquant où elle voulait son église. Ibid., 92.

61. Ibid., 90-91. Les sœurs Gros-Louis appuient ce récit sur des histoires orales huronnes. Comme c'est souvent le cas avec la tradition orale, le temps est aboli. Les histoires tournent autour de 
Dans ce chassé-croisé de reliques, il est intéressant de noter ce que les Hurons de la Jeune-Lorette ont conservé : le bas-relief en bois de la Santa Casa d'Italie, cette maison qui était tombée du ciel ${ }^{62}$. Il y avait peut-être une certaine coïncidence entre cette chute cosmogonique et le récit huron des origines qui raconte la chute du ciel d'Aataentsic, la mère de l'humanité . Cependant, à la mission de la Jeune-Lorette, la Sainte Maison évoquait autre chose que les apparitions mythiques d'Aataentsic ou de la miraculeuse relique mariale d'Italie. Tout comme l'ajout d'un nom, l'ajout d'une maison concrète à l'histoire du commencement d'une collectivité est significatif. La Sainte Maison importée était évidemment devenue un important symbole personnel pour ces Hurons convertis. Elle évoquait peut-être leur expérience récente d'une chapelle jésuite et d'un chez-soi catholique où certains d'entre eux s'étaient sentis en sécurité. Même lorsque ces Hurons furent écartés par un groupe de catholiques français, la reproduction en bois de la Santa Casa indiquait qu'ils avaient été accueillis par d'autres catholiques, les Jésuites.

Peut-être pour honorer cette parenté catholique et cette nouvelle identité, les Hurons ont placé la maison en bois bien en évidence dans leur nouvelle église ${ }^{63}$. Elle s'y trouve toujours, au centre du chœur et au-dessus de l'autel dans l'église de la réserve de Wendake, près de Loretteville, au Québec. Cette sculpture revêt aujourd'hui une signification additionnelle, car il s'agit de la seule pièce provenant de la mission de Lorette que les Hurons possèdent; le reste des ornements ont disparu dans un incendie. Elle retient aussi l'attention parce que, si l'église huronne a été rénovée pour faire place à de nombreux articles importants pour les Hurons sur

l'intervention de $\mathrm{M}^{\mathrm{gr}}$ de Laval, évêque de Québec, mais il avait démissionné de ses fonctions une bonne dizaine d'années avant que les Hurons déménagent à la Jeune-Lorette.

62. Des objets de piété autres que des statues voyageaient aussi, à cette époque: ils franchissaient l'Atlantique dans un sens comme dans l'autre. Il y a un intéressant corollaire à cet épisode de Québec: il s'agit de l'échange de plaques de la basilique et de ceintures de wampum entre Loreto, en Italie, et les Hurons de la Jeune-Lorette. À propos des plaques, voir Lionel Saint-George Lindsay, Notre-Dame de la Jeune-Lorette en la Nouvelle-France. Étude historique (Montréal, 1900), 186-187. Sur les ceintures de wampum que les Hurons de la Jeune-Lorette ont envoyées, non seulement à Loreto, mais aussi à Chartres et à d'autres lieux de pèlerinage en Europe, voir André Sanfaçon, "Objets Porteurs d’identité dans les consécrations amérindiennes à Notre-Dame de Chartres, 1678-1749", dans Laurier Turgeon, Denys Delâge et Réal Ouellet, dir., Transferts culturels et métissages. Amérique/Europe XVI ${ }^{e} X^{e}$ siècle/Cultural Transfer, America and Europe: 500 Years of Interculturation (Sainte-Foy, Les Presses de l'Université Laval, 1996), 449466. Voir aussi Jonathan Christopher Lainey, La "monnaie des sauvages». Les colliers de wampum d'hier d̀ aujourd'hui (Sillery, Septentrion, 2004), 106; Karin Vélez, "A Sign That We Are Related to You”: The Transatlantic Gifts of the Hurons of the Jesuit Mission of Lorette, 1650-1750 ", French Colonial History, 12 (2011): 31-44.

63. Visite guidée de la chute Kabir Kouba et de l'église Notre-Dame-de-Lorette, organisée par la Maison Arouanne, Conseil de la Nation Huronne-Wendat, Wendake (Québec), juin 2006. 


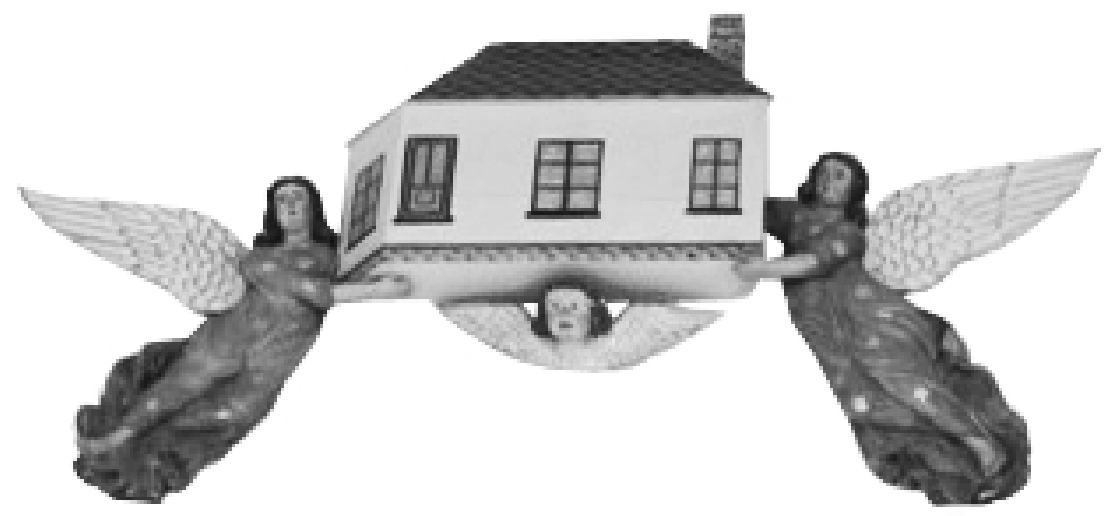

Bas-relief en bois $\left(17^{\mathrm{e}} \mathrm{s}\right.$.) de la Santa Casa de Lorette, église de Notre-Dame-de-Lorette, Wendake, Québec

Photo: Thomas Wien, 2006

le plan culturel, comme des raquettes et des œuvres brodées et ornées de perles, la sculpture est l'un des très rares objets de provenance incontestablement française qui sont exposés dans la nef. La sculpture date du XVII ${ }^{e}$ siècle et est attribuée à l'atelier de la famille Levasseur, de Québec ${ }^{64}$. La maison sculptée a été modifiée avec le temps: elle avait trois fenêtres, mais elle en a quatre aujourd'hui. Elle se rapproche de l'architecture française ordinaire du XVII ${ }^{e}$ siècle plus qu'à la Sainte Maison en Italie. Le bas-relief comporte aussi trois anges blancs, deux sur les côtés et un en bas, qui transportent la maison; Marie n'y est pas représentée ${ }^{65}$.

L'œuvre d'art encore exposée à Wendake appartient à une longue tradition catholique, commune à tout le monde atlantique, de reliques qui déménagent. Des histoires concernant des objets sacrés qui se déplacent de leur propre gré étaient courantes dans le monde atlantique de l'époque moderne, partout où on se disputait la propriété de reliques catholiques ${ }^{66}$.

64. Raymonde Gauthier, «Un art de vivre et de créer: la dynastie des Levasseur», Critère, 12 (1975): $127-139$.

65. C. Gros-Louis et C. Gros-Louis, La Chapelle huronne de Lorette..., op. cit., 61-62.

66. Ces phénomènes semblent survenir purement dans des situations de discorde. En 1708, en France, par exemple, une statue miraculeuse déménagea d'une petite chapelle rurale, Notre-Dame-de-Lorettesur-le-Mont, à la ville de Gondrecourt. Même si des pèlerins avaient défilé devant la statue au premier lieu, l'église où elle se trouva ensuite annonça tout simplement aux pèlerins intéressés qu'ils devraient désormais se rendre à Gondrecourt, et l'affaire fut réglée sans autre envol de reliques ni intervention épiscopale. Voir Philippe Martin. "Une pastorale pèlerine dans la Lorraine du XVIII siècle», dans Phillipe Boutry, Pierre-Antoine Fabre et Dominique Julia, dir., Rendre ses vœux. Les identités pèlerines dans l'Europe moderne (XVI - XVIII ${ }^{e}$ siècle) (Paris, École des hautes études en sciences sociales, 2000), 537. 
Ce qui est fascinant à propos de cette variante huronne des reliques voyageuses, cependant, c'est l'autodétermination huronne. Les Hurons de la Jeune-Lorette expliquent eux-mêmes le déplacement de la relique d'une manière catholique, avec des noms et des symboles catholiques. Dans l'histoire qu'ils racontent, le conflit, la négociation et la proximité étroite avec les Français ont consolidé l'identité catholique des Hurons. Ils se sont engagés auprès des colons de Québec en tant que coreligionnaires catholiques. Les fidèles hurons et les fidèles français voulaient des objets de la vieille église des Jésuites; les uns et les autres voulaient les objets sacrés qui se trouvaient en son centre, les uns et les autres ont écouté l'évêque d'une oreille distraite; les uns et les autres sont sortis de là avec un élément précieux de la chapelle qu'ils avaient eue en commun; et les uns et les autres ont conservé le nom de Loreto donné par les Jésuites.

\section{CONCLUSION}

En conclusion, cette histoire orale huronne du déplacement autopropulsé de reliques lorettaines souligne comment les Hurons ont revendiqué le nom et les ornements de Loreto. Leur revendication soutient la comparaison avec celle des Italiens qui, des siècles plus tôt, ont accueilli un objet étranger sur leurs rives en lui donnant un nom local: "Laureta». Même si le moyen choisi par les Italiens était exactement à l'opposé de celui choisi par les Hurons, soit de donner une étiquette locale à ce qui vient de loin plutôt que de donner un nom étranger à ce qui est local, il y eut le même élan pour s'approprier un nom qui s'imposait dans les deux cas. Enfin, au XVIII ${ }^{\text {e }}$ siècle, nous pouvons reconnaître plus nettement les acteurs qu'au XIII ${ }^{\text {: }}$ les Jésuites, les Hurons et les colons français de Québec, qui figurent ici non comme des personnes voulant imposer un nom, mais comme des frères et des sœurs dans le catholicisme. Ces trois groupes de fidèles se sont rassemblés autour d'objets sacrés et d'un nom - Loreto/Jeune-Lorette/ Ancienne-Lorette; un nom ancien, puis nouveau et redevenu ancien.

Dans le présent article, nous avons suivi le nom de Loreto à partir du sommet d'une colline italienne jusqu'à des ports de mer de l'Atlantique, depuis les avant-postes ruraux de la Nouvelle-Espagne jusqu'aux forêts nordiques des Hurons. Il est clair que le caractère multidimensionnel des noms a permis à des Jésuites comme Chaumonot d'assumer et d'imposer Loreto, mais aussi de l'inviter et de le laisser partir outre-mer, où de nouveaux peuples en ont pris possession. Dans le monde atlantique du XVII ${ }^{e}$ siècle, les noms étaient souvent accueillis comme de nouvelles couches 
d'identité, et comme des témoins de nouveaux liens entre des peuples. Comme le démontre le cas des Hurons de la Jeune-Lorette, les noms n'étaient pas seulement attribués dans cette zone de rencontre culturelle. Les noms étaient revendiqués et gardés. C'est ainsi que Loreto - qui fut d'abord un nom local discret - finit par faire une bonne partie du travail de propagation d'un élément du catholicisme. Ce nom étendit en effet le «territoire de reconnaissance et de dévotion» de ce culte marial, depuis son circuit étroit, foulé par les pèlerins en Italie, à l'Amérique autochtone et au Canada français, et ensuite dans l'ensemble du monde atlantique du XVII ${ }^{\mathrm{e}}$ siècle $^{67}$.

Traduction: Hélène Paré

67. William Taylor en dit autant de la grande popularité de la Vierge de Guadalupe au Mexique. Voir William B. Taylor, «Dos Palabras on Mexican Shrines and Contagiousness of the Sacred», communication devant la Conference on Latin American History dans le cadre du congrès de l'American Historical Association, Washington, 4 janvier 2008, 8. 